\title{
Gestion Del Conocimiento En Universidades Públicas Mexicanas
}

\author{
Dra. Ana Laura Luna Jiménez. \\ Universidad Juárez Autónoma de Tabasco, México \\ Dr. Roberto Reyes Cornelio \\ Universidad Juárez Autónoma de Tabasco, México \\ M en C. Ygnacia Jiménez Vera \\ Instituto Tecnológico de Mérida, Yucatán, México
}

\section{doi: 10.19044/esj.2017.v13n1p54 $\quad$ URL:http://dx.doi.org/10.19044/esj.2017.v13n1p54}

\begin{abstract}
Public universities in Southeast of Mexico need to increase their scientific productivity to generate competitive advantages that increase their economic value. The academic ability indicators that measure the knowledge management of these institutions are below the national average. The purpose of this research was to analyze the knowledge management in the public universities in Southeast Mexico as a tool to increase their competitiveness. Interviews and surveys were applied to managers, middle managers and teachers. It was found that in these universities the knowledge factor is not managed efficiently. This research provides information on the current state of knowledge management and a knowledge management model for these educational institutions. The model will enable the implementation of efficient knowledge management and the development of its intellectual capital.
\end{abstract}

Keywords: Knowledge management, university, knowledge, intellectual capital

\section{Resumen}

Las universidades públicas estatales del sureste de México necesitan incrementar su productividad científica para generar ventajas competitivas que incrementen su valor económico. Los indicadores de capacidad académica, que miden la gestión del conocimiento de estas instituciones están por debajo de la media nacional. El objetivo de esta investigación fue analizar la gestión del conocimiento en las universidades públicas del sureste de México como herramienta para elevar su competitividad. Se realizaron entrevistas y encuestas a directivos, mandos medios y docentes. Se encontró que en estas universidades el factor conocimiento no se gestiona de forma eficiente. Esta 
investigación aporta información sobre el estado actual de la gestión del conocimiento y un modelo de gestión del conocimiento para estas instituciones educativas. El modelo permitirá implementar una gestión eficiente del conocimiento y el desarrollo de su capital intelectual.

Palabras clave: Gestión del conocimiento, universidad, conocimiento, capital intelectual

\section{Introducción}

La gestión del conocimiento como cualquier área del saber y producción humana no tiene una fecha de inicio exacta. De acuerdo con Montiel (2015), existen dos posiciones extremas que determinan su inicio. Una de ellas afirma que surgió desde el primer momento en que nació el conocimiento, mientras que la otra indica que fue en los años noventa, con la obra "The Knowledge-Creating Company" de Nonaka y Takeuchi, en que la gestión del conocimiento ganó prominencia como una aproximación y redirección de energías y actividades para la generación, flujo y uso del conocimiento interno.

La gestión del conocimiento ha sido estudiada como técnica de gestión cuyo objetivo es maximizar la aportación de las organizaciones a la sociedad. A partir de los cambios provocados por la globalización de la economía, se hace evidente que las universidades, al ser formadoras de capital humano y generadoras de conocimiento a través de la investigación, necesitan gestionar estos recursos para sobrevivir en un medio ambiente competitivo (Blázquez, 2011).

Las universidades, por la naturaleza de sus funciones, son organizaciones gestoras del conocimiento, aunque esto sólo les da una ventaja inicial (Olvera y Morales, 2011). Las universidades públicas en México, son instituciones dedicadas a la generación y aplicación del conocimiento, sin embargo, no se ha realizado una valoración de sus contribuciones a la sociedad, medida por su valor de mercado. En un futuro, las universidades públicas en México, tendrán que determinar su valor incluyendo sus activos intangibles y fuente de ventajas competitivas, para estas instituciones.

Por otra parte, las universidades públicas en México, enfrentan cada vez más presiones financieras. Estas instituciones tienen que demostrar un mayor nivel de competitividad, a través de reportes de resultados basados en su capital intelectual, lo que las pone en desventaja con otras instituciones como la Universidad Nacional Autónoma de México (UNAM), que reciben mayores recursos del gobierno (Villaseñor, Moreno y Flores, 2015).

El objeto de estudio de esta investigación fueron las universidades públicas estatales de la región sur sureste de la ANUIES que abarca las universidades de: Campeche, Ciudad del Carmen, Chiapas, Oaxaca, Quintana 
Roo, Tabasco, Veracruz y Yucatán, debido a que registran un bajo desarrollo de los indicadores de su capital intelectual. La Secretaría de Educación Pública (SEP) en México, mide la capacidad académica de las instituciones de educación superior a través de los indicadores del capital intelectual que generan y de acuerdo a ello otorga recursos adicionales al presupuesto asignado y mide la competitividad de las universidades.

Lo anterior, ha ocasionado que las universidades se interesen en incrementar sus indicadores de productividad. Los directivos de estas universidades, necesitan gestionar con mayor eficiencia el conocimiento para incrementar su productividad y ser competitivas. El conocimiento es un factor más de la producción que hay que explotar en beneficio de la institución, pero para ello se necesita incrementar sus indicadores de productividad.

Desde esta perspectiva se justifica un cambio de gestión en las organizaciones que se oriente a la creación de valor. La nueva gestión debe de estar orientada al conocimiento y a la generación de valores basados en intangibles. La dirección de estas universidades debe asegurar altos niveles de calidad, que contribuyan a la generación de valor de mercado en estas organizaciones para justificar el presupuesto que el gobierno les asigna.

Si se realiza un análisis desde el punto de vista económico-financiero, el valor contable de la universidad es la suma del valor de sus activos tangibles (edificios, equipos de laboratorio, entre otros). Sin embargo, el valor de los intangibles, quienes son los que en realidad aportan valor a las organizaciones, están ausentes por lo general en los estados financieros especialmente en aquellas organizaciones que son intensivas en la creación y difusión del conocimiento como las universidades (Rivero, Vega y Balagué, 2005).

A partir de este razonamiento, y teniendo presente la contribución que tienen las universidades en la producción científica de un país, se hace necesario proponer técnicas de gestión que mejoren la aportación de las universidades a la sociedad.

Si la educación pública de nivel superior, así como muchos otros servicios que brinda el gobierno informaran su costo real a la sociedad, entonces, sería imprescindible para las universidades generar informes financieros, incluyendo indicadores no financieros. Esto les concedería un mayor grado de importancia a estas instituciones, porque su valor económico se justificaría en tanto su aportación al desarrollo económico del país.

Se espera que, en un futuro las universidades públicas, tendrán que elaborar informes con indicadores financieros y no financieros, donde demuestren a través de resultados su competitividad para justificar los recursos recibidos y se pueda comparar su valor generado con el de otras instituciones de educación superior. De ahí, la relevancia de este trabajo, que aporta información sobre los avances de las universidades de la región sur sureste en el tema de la gestión del conocimiento. 
En México se han realizado estudios encaminados a la gestión del conocimiento y capital intelectual en universidades públicas. Topete y Bustos (2008), investigadores del Instituto Politécnico Nacional (IPN) identificaron estrategias para la planeación en instituciones de educación superior. Encontraron las mejores prácticas sobre la gestión del capital intelectual en el sector universitario.

En el sureste del país, Barroso (2011), investigó la gestión del conocimiento en once Instituciones de Educación Superior (IES) y Centros de Investigación Científica (CIC's) en el estado de Yucatán. Se encontró que es necesario estas instituciones cuenten con más recursos y programas de vinculación entre IES, empresas, gobierno y CIC's, que permitan procesos adecuados de gestión del conocimiento (Barroso, 2011).

Mijangos y Manzo (2012), estudiaron la gestión del conocimiento en cuerpos académicos de universidades públicas de estados ubicados al sur y sureste de México. Sus resultados indican que la gestión del conocimiento al interior de los cuerpos académicos, se realiza a través de estrategias que pudieran replicarse en los procesos de gestión del conocimiento de otros cuerpos académicos. Asimismo, se recomienda el estudio de los vínculos entre el tipo de liderazgo y la gestión del conocimiento.

Por su parte, Magaña, Aguilar, Surdez y Quijano (2013), investigaron la gestión del conocimiento de los cuerpos académicos en la Universidad Juárez Autónoma de Tabasco (UJAT). Sus hallazgos confirmaron la necesidad de adecuar la estructura organizacional de esta institución, para un funcionamiento óptimo de los procesos de gestión del conocimiento al interior de los cuerpos académicos y que el conocimiento sea generado a través de las funciones sustantivas de investigación, docencia y vinculación.

En los estados del sureste de México el mayor número de investigaciones relacionadas con la gestión del conocimiento son dirigidas al sector empresarial y pocas a instituciones educativas, se hace latente la necesidad de nuevas investigaciones en el contexto de la educación superior en esta región.

Los directivos de las organizaciones y las instituciones educativas tienen que aumentar el valor de sus activos intangibles, para darles mayor valor económico y social a sus instituciones. Es pertinente, que además de tener la información financiera y contable para rendir cuentas a la sociedad, se elaboren reportes de capital intelectual que complementen y actualicen el valor económico de la institución.

En esta investigación, se puntualiza la necesidad e importancia de la gestión del conocimiento, como fuente de ventajas competitivas en las organizaciones de todo tipo. Una aplicación efectiva de la gestión del conocimiento demanda cambios en el proceso de gestión del mismo, así como la necesidad de adecuar las estructuras organizativas y los estilos de liderazgo. 


\section{Metodología}

El alcance de esta investigación fue exploratorio-descriptivo con un diseño metodológico mixto. El diseño mixto utilizado contempló dos fases. La fase I respondió a un enfoque cuantitativo al determinar resultados numéricos utilizando la técnica de la encuesta a directivos y mandos medios de las universidades estudiadas. La fase II, dentro de un enfoque cualitativo, se utilizó la técnica de la entrevista a docentes, para ofrecer mayor sentido a los datos numéricos y obtener evidencias y comprensión de la gestión de conocimiento en las universidades estudiadas. Esta segunda fase se nutrió de la fase I para su diseño y desarrollo.

El universo de trabajo se constituyó por el sistema de universidades públicas de las entidades federativas de México que, en 2015, de acuerdo con la información de la SEP son 34 instituciones. Para realizar la investigación empírica fue necesario efectuar una selección de la muestra por conveniencia.

La selección de la muestra se realizó listando todas las universidades públicas de la región sur sureste de la Asociación Nacional de Universidades e Instituciones de Educación Superior (ANUIES). Se tomaron como muestra ocho universidades públicas de la región sur sureste de la ANUIES que se caracterizan por tener en común indicadores de capital intelectual no satisfactorios descritos en el capítulo uno de esta investigación (Tabla 1).

Tabla 1. Universidades región sur sureste de la ANUIES

\begin{tabular}{cc}
\hline Número & Universidad \\
\hline 1 & Universidad Veracruzana \\
2 & Universidad Juárez Autónoma de Tabasco \\
3 & Universidad Autónoma de Chiapas \\
4 & Universidad Autónoma Benito Juárez de Oaxaca \\
5 & Universidad Autónoma de Yucatán \\
6 & Universidad Autónoma de Campeche \\
7 & Universidad Autónoma del Carmen \\
8 & Universidad de Quintana Roo \\
\hline
\end{tabular}

Posteriormente, en función de los recursos para realizar esta investigación, se realizó un segundo muestreo limitándose a doce escuelas relacionadas con el área de negocios de las universidades públicas seleccionadas. En función de los objetivos de esta investigación, la población sujeto de estudio fueron 113 directivos y mandos medios de estas instituciones. En función del tamaño pequeño de la muestra se utilizó el censo.

La unidad de análisis, estuvo constituida por los directivos y mandos medios de estas instituciones que conforman la alta dirección de las mismas y de quien depende se lleve a cabo una eficiente gestión del conocimiento. 
Para recabar información se utilizó el cuestionario, consulta de documentos obtenidos de Internet y de publicaciones tanto internas como externas a las universidades objeto de estudio (páginas web, documentos institucionales y bases de datos). El cuestionario se integró por tres secciones de gestión del conocimiento, dirección y capital intelectual, con escala de Likert de valoración del 1 al 5.

Se realizó una prueba de confiabilidad para el instrumento mediante la determinación del coeficiente Alfa de Cronbach a fin de validar y probar la consistencia del instrumento. El coeficiente tuvo un valor global de 0.853 lo que garantiza una buena fiabilidad. El instrumento utilizado en esta investigación fue validado utilizando tres tipos de evidencia: validez de contenido, de expertos y de constructo.

El cuestionario se aplicó directamente y por correo electrónico a directivos y a los responsables de mandos medios de las universidades seleccionadas en el muestreo. Para complementar la información, se realizaron entrevistas a docentes de las universidades objeto de estudio. Las entrevistas realizadas estuvieron caracterizadas por ser de tipo individual directa entre entrevistador y entrevistado y de no dirección o semiestructurada con un guion orientado a preguntas abiertas.

\section{Resultados}

El análisis realizado al estado de la gestión del conocimiento en ocho escuelas de negocios de universidades públicas de la región sureste de México permitió obtener los siguientes resultados.

\section{Dimensión el conocimiento como factor estratégico}

El análisis de la dimensión del conocimiento como factor estratégico, tuvo como finalidad evaluar el conocimiento como factor estratégico en las universidades públicas mexicanas y conocer la forma en que se lleva a cabo su proceso económico.

En estas universidades se reconoce que el conocimiento es un factor estratégico para elevar la productividad del trabajo docente, así lo confirman los resultados de esta investigación $(62 \%)$ de percepción favorable. No obstante, los resultados de indicadores claves de la gestión del conocimiento en estas instituciones: cuerpos académicos en formación y consolidación, docentes con perfil PRODEP, número de investigadores (SNI) y número de publicaciones demuestran que no se tiene una gestión del conocimiento planificada y eficiente.

El número de cuerpos académicos formados en las universidades objeto de estudio se encuentra por debajo de la media nacional, que corresponde al $28.71 \%$ en lo que se refiere a cuerpos académicos en 
consolidación y del 22.97 \% de cuerpos académicos consolidados (SEP, 2013) (Figura 1).

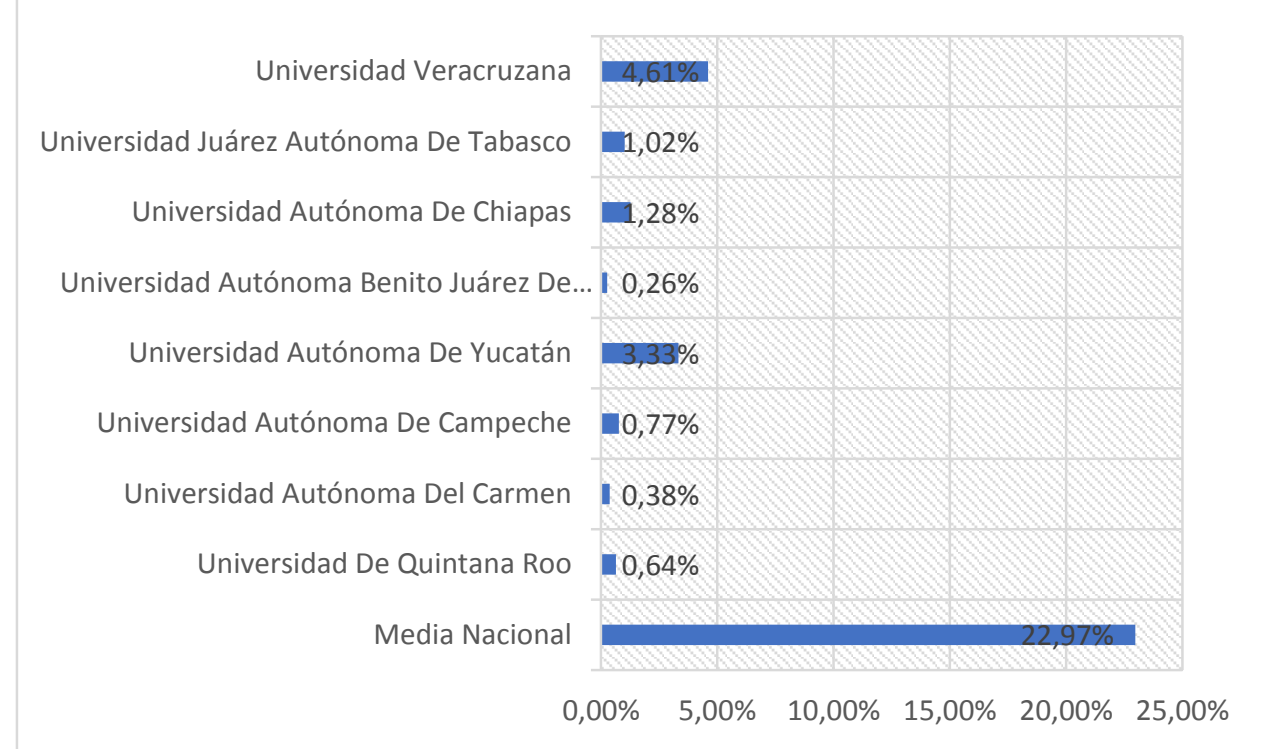

Figura 1. Cuerpos académicos consolidados.

Fuente: Fuente: Secretaría de Educación Pública, 2013.

Este indicador desfavorable se debe, de acuerdo a los docentes entrevistados, a la falta de acompañamiento en el proceso de conformación de cuerpos académicos así como a una baja motivación para integrarlos. Los entrevistados, también expresaron una falta de apoyo para realizar proyectos, producir conocimiento e intercambiarlos, además, no consideran favorable las condiciones para la conformación de cuerpos académicos en su institución. Por lo anterior, se considera necesario mejorar el proceso económico del conocimiento en estas universidades.

En cuanto al indicador de profesores con perfil PRODEP, sólo es satisfactorio en dos universidades: la Universidad de Quintana Roo y la Universidad Autónoma de Yucatán. Las otras universidades estudiadas no superan la media nacional del $58.34 \%$ (SEP, 2013) (Figura 2). 


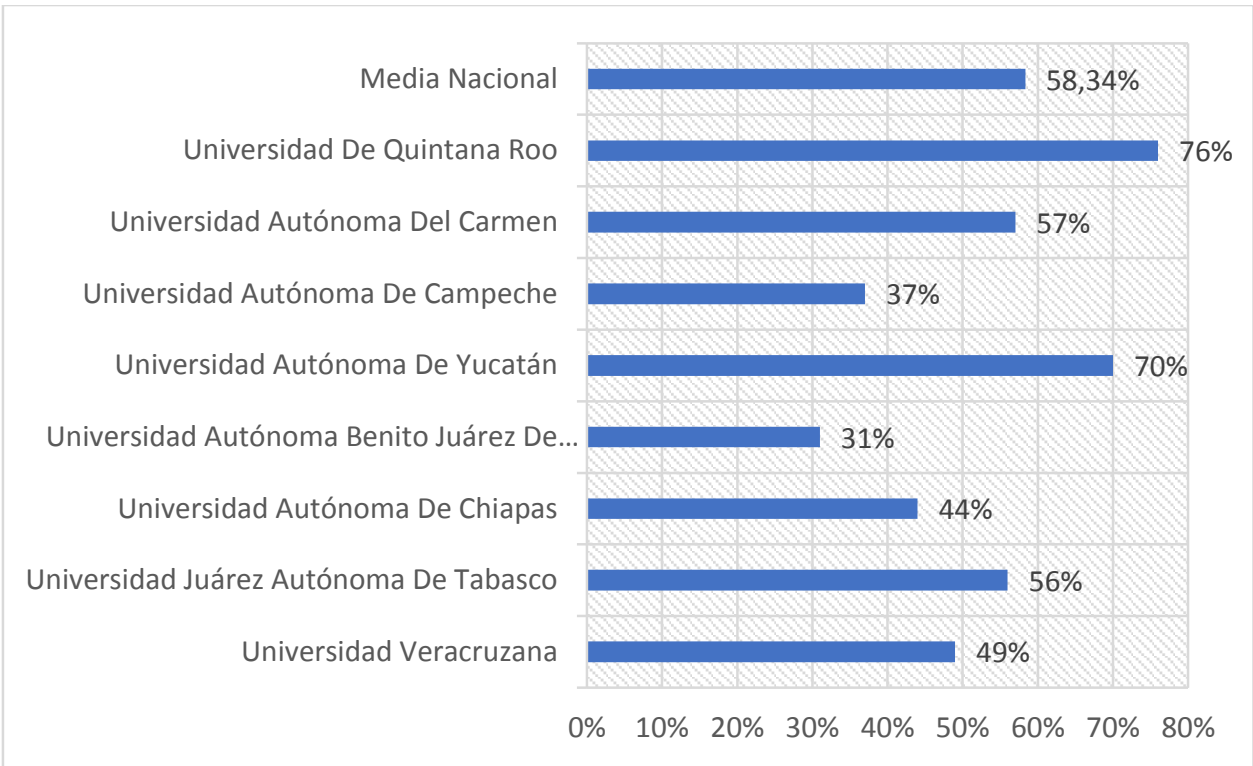

Figura 2. Profesores con perfil PRODEP.

Fuente: SEP, 2013.

En relación a este indicador, los docentes entrevistados no perciben prioridad por parte de los directivos para realizar las actividades que se les solicita para obtener el perfil PRODEP.

Lo anterior, también se aplica a los investigadores del Sistema Nacional de Investigadores (SIN) ya que siete de las universidades objeto de estudio están por debajo de la media nacional 17 \% (ExeCum, 2014) (Figura $3)$.

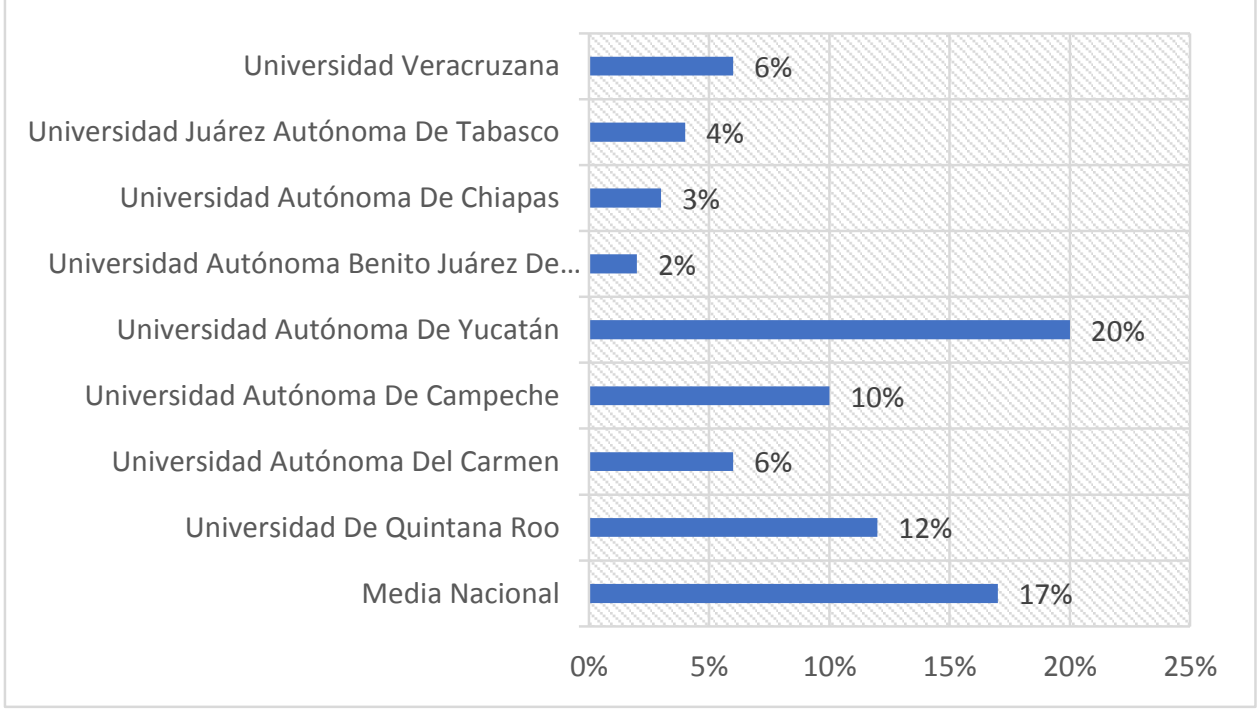

Figura 3. Profesores adscritos al Sistema Nacional de Investigadores Fuente: Execum, 2014. 
En opinión de los docentes entrevistados, los programas antes mencionados no son capaces de motivar y propiciar una mayor producción e intercambio sobre todo del conocimiento, lo limitan, ya que la generación del conocimiento debe de ser una forma de vida en estas instituciones. Es decir, el docente que desee participar en proyectos innovadores, se le debe dar la oportunidad y el estímulo, independientemente de contar con una plaza de tiempo completo.

La investigación aplicada y la formación de equipos de trabajo no es significativa en las instituciones objeto de estudio. No se aprovecha eficientemente la conversión del conocimiento que puede darse cuando estas organizaciones interactúan con el medio ambiente, ya sea como generadoras o receptoras de conocimiento. En consecuencia, no se documenta, almacena y dispone del conocimiento que se genera, día con día, en sus funciones sustantivas.

En opinión del $55 \%$ de los directivos encuestados, la investigación aplicada se realiza en forma colegiada a través de proyectos innovadores, lo que indica que es factible implementar proyectos de gestión que generen valor a la institución. Por lo que es de suma importancia, la implementación de la planeación estratégica del conocimiento en las universidades estudiadas.

Es importante mencionar, que la producción, intercambio y aplicación del conocimiento tiene alcance local, ya que $72 \%$ de los directivos entrevistados están en desacuerdo en que el conocimiento generado tenga alcance internacional. De acuerdo con entrevistas a docentes, los apoyos otorgados son insuficientes para la producción de conocimientos de calidad, lo que impide que estas instituciones sean competitivas a nivel internacional. Además, no se promueve una cultura que genere conocimientos para compartirlo, al menos entre los investigadores de las universidades y otras instituciones.

En cuanto al impacto en el desarrollo tecnológico, de acuerdo con los resultados obtenidos, en la producción de conocimientos se favorece a la tecnología de valor de cambio. Por ello, se infiere que en las instituciones analizadas, no se reconoce la necesidad de generar tecnologías de valor de uso. Las tecnologías de valor de uso, tienen ventajas sobre las de valor de cambio ya que su financiamiento, con frecuencia, suele ser más económico y resultan ser fuentes de capital intelectual, porque contribuyen a la generación de: patentes, sistemas, modelos, técnicas administrativas, entre otras.

\section{Dimensión dirección estratégica del conocimiento}

La segunda dimensión analizada, fue la dirección estratégica del conocimiento con el objetivo de identificar si la función directiva se realiza con eficiencia y eficacia en el logro de un liderazgo transformacional, una comunicación estratégica corporativa, planeación estratégica del 
conocimiento, así como la creación de ventajas competitivas. Se analizaron el liderazgo, el trabajo en equipo, la comunicación estratégica, la satisfacción en el trabajo, la planeación y dirección estratégica del conocimiento.

Los estilos de liderazgo representan un factor de éxito en la gestión del conocimiento $\mathrm{y}$, por ende, en la competitividad de las organizaciones (Rodríguez-Ponce, Pedraza-Rejas, Delgado y Rodríguez-Ponce, 2010). La percepción del estilo de liderazgo, en los encuestados de las universidades es negativa, ya que sólo un $28 \%$ de los directivos considera que se ejerce un liderazgo participativo basado en resultados, lo que implica la necesidad de cambio en los estilos de liderazgo de estas instituciones para gestionar el conocimiento, que demanda un tipo de liderazgo transformacional para que el conocimiento se direccione de forma adecuada.

El trabajo en equipo presentó una percepción favorable, ya que el 90 $\%$ de los encuestados están de acuerdo que en su institución se fomenta el trabajo en equipo, lo que facilita en estas instituciones la formación de equipos de alto desempeño, así como la creación de grupos académicos que busquen la asociación para generar conocimientos.

La comunicación estratégica hacia el interior de la organización, los resultados obtenidos indican que en las universidades motivo de estudio se da una comunicación de manera vertical que obstaculiza la socialización, el intercambio y aplicación del conocimiento, característicos de una estructura organizativa burocrática.

La comunicación estratégica con externos como son: centros de investigación, empresas y gobierno, la percepción es baja (33\%) ya que los encuestados perciben que la vinculación no se ha incrementado. Al respecto, los docentes entrevistados expresaron que existen pocos mecanismos en sus instituciones que permitan la comunicación con el exterior. Por ello, se deduce que esta comunicación no es efectiva, lo que representa un área de oportunidad para la alta dirección de estas instituciones.

Con relación a la satisfacción en el trabajo, se encontró una percepción positiva, ya que el $59 \%$ de los encuestados están de acuerdo y $17 \%$ muy de acuerdo en que las estrategias implementadas para gestionar el capital intelectual han incrementado la satisfacción del trabajo en estas instituciones. Por lo que se debe de continuar con el fomento de programas que incentiven la generación de capital intelectual.

En cuanto a la planeación y dirección estratégica del conocimiento, los resultados indican que son desfavorables, ya que el $59 \%$ de los encuestados opinan que la planeación estratégica del conocimiento no se lleva a cabo. De acuerdo con los docentes entrevistados, la planeación estratégica en estas universidades se realizada de acuerdo con el Programa Integral de Fortalecimiento Institucional (PIFI) y en esta planeación, no se incluye la gestión del conocimiento. 
Para que la dirección estratégica del conocimiento de estas instituciones pueda mejorarse resulta fundamental que se incluya en la planeación estratégica un proceso para compartir el conocimiento adquirido por los miembros de la organización (Bustos, et al. 2014). En este sentido, Núñez y Rodríguez (2015) afirman que existe una relación directa entre la gestión del conocimiento y la gestión estratégica de las IES. Destacan además, el hecho que la gestión estratégica de las IES puede mejorarse a través de la implementación de los procesos de la gestión del conocimiento y del capital intelectual.

\section{Dimensión el capital intelectual}

Esta dimensión tuvo como objetivo el estudio de los elementos estructurales, relacionales y humanos del capital intelectual que integran el capital intelectual en estas universidades. Las variables analizadas, fueron competencias del personal, certificaciones, datos patentados, sistema de indicadores del capital intelectual, clima laboral, comunidades y redes, plataformas de base de datos y sistemas de información.

Con relación a la valoración del capital humano en estas instituciones, los encuestados presentaron una percepción favorable sobre la capacidad del análisis de las personas contratadas (69 \%). En la mayoría de estas instituciones, se exige como requisito la preparación y experiencia al contratar personal, lo que representa una fortaleza, al contar con personal capacitado.

Esta condición se considera un indicador de importancia en las instituciones encuestadas, de acuerdo con Núñez, Arutimuño y Rodríguez (2014), las IES que cuentan con individuos que tienen conocimientos de alto valor potencializan los procesos de gestión de conocimiento y los intangibles.

Una de las variables de mayor impacto para el capital estructural son los sistemas tecnológicos, de acuerdo con Peñaloza, Arévalo y Pirela (2010) la gestión del capital intelectual no significa únicamente adquirir y acumular conocimientos, sino ser capaz de transformarse y diseminarse en toda la organización. Para esto se requiere infraestructura tecnológica y sistemas de información como: bases de datos inteligentes, herramientas para la captura de datos, redes de comunicación, herramientas de colaboración, intranets, entre otros, que permitan la adquisición, compartimiento y utilización del conocimiento.

La percepción de los directivos sobre la gestión del capital estructural indica que un pequeño porcentaje, el $24 \%$ de las universidades, cuentan con plataforma de base de datos que permiten el aprendizaje organizacional, lo que indica que es necesario promover el uso de las tecnologías de la información y la comunicación, así como el uso de herramientas colaborativas.

En la gestión del capital relacional, las variables analizadas fueron comunidades y redes de aprendizaje, mecanismos de opinión y valor agregado 
a usuarios, los resultados no son favorables, ya que los directivos encuestados perciben que no se fomenta este tipo de prácticas en la institución, por lo que es necesario, el incremento del número de convenios de colaboración para establecer mayores vínculos con el sector productivo.

Asimismo, la variable sobre la existencia de mecanismos de opinión del sector empresarial, gobierno y público en general acerca de los servicios que ofrecen estas instituciones es desfavorable. Además, se desconoce si en las universidades estudiadas el presupuesto para la difusión y promoción de los servicios que prestan estás instituciones es suficiente, para crear un buen posicionamiento en el mercado educativo.

Se concluye que, aunque existe potencial para gestionar eficientemente el conocimiento e incrementar su capital intelectual, estas universidades siguen trabajando de manera tradicional, no tienen un inventario de sus activos intangibles, por lo que su generación es fortuita.

Las universidades públicas estatales necesitan demostrar que son instituciones valiosas en lo económico y social, por lo que es necesario cuantificar sus activos tangibles e intangibles para generar un valor de mercado con indicadores financieros y no financieros, que permitan medir su capacidad económica y competitividad educativa $y$ poder hacer comparaciones con otras universidades, ya sean nacionales o internacionales, tomando en cuenta la riqueza que estas instituciones pueden aportar a la sociedad.

\section{Modelo propuesto:}

Para gestionar eficientemente el conocimiento en las universidades públicas de la región sur sureste de México para que eleven su productividad y generen ventajas competitivas que incrementen su valor de mercado se necesita la implementación de un modelo de gestión del conocimiento que incremente el capital intelectual de estas instituciones, basado en un proceso económico del conocimiento y una dirección estratégica del mismo.

\section{Desarrollo del modelo de gestión del conocimiento para universidades públicas estatales}

El desarrollo del modelo de gestión del conocimiento para una institución de educación superior se explica de acuerdo con el enfoque de sistemas o sistémico, con un objetivo así como la correspondiente entrada, proceso y salida del modelo

Objetivo. Gestionar eficientemente el conocimiento para incrementar el valor de mercado de estas instituciones mediante la creación de ventajas competitivas proveniente de su capital intelectual. 
Entrada. Se considera toda aquella información necesaria para que los tomadores de decisiones de la alta dirección de las mencionadas instituciones puedan gestionar eficiente y eficazmente el conocimiento como son:

- Identificación del conocimiento y detección de necesidades del conocimiento.

- Identificación del proceso económico del conocimiento (producción, distribución, intercambio y consumo) que una buena gestión gerencial del conocimiento exige.

Proceso de transformación. Representa los cambios que la alta dirección debe hacer para sentar las bases de una gestión gerencial basada en el conocimiento como son:

- Una estructura organizativa horizontal, democrática flexible y por procesos, en vez de una estructura burocrática,

- Un liderazgo transformacional en vez de un liderazgo autocrático,

- La implementación de una planeación estratégica del conocimiento que contribuya para planear e implementar una gestión efectiva, como una herramienta de planeación y de control del proceso de gestión del conocimiento.

- Buen posicionamiento en el mercado de la educación superior.

Salida. Representa los resultados del proceso de gestión del conocimiento considerados como capital intelectual en sus tres dimensiones:

- Capital humano,

- Capital estructural y

- Capital relacional.

Etapas del modelo. El modelo de gestión del conocimiento para universidades públicas estatales consta para su implementación de tres etapas (Figura 4)
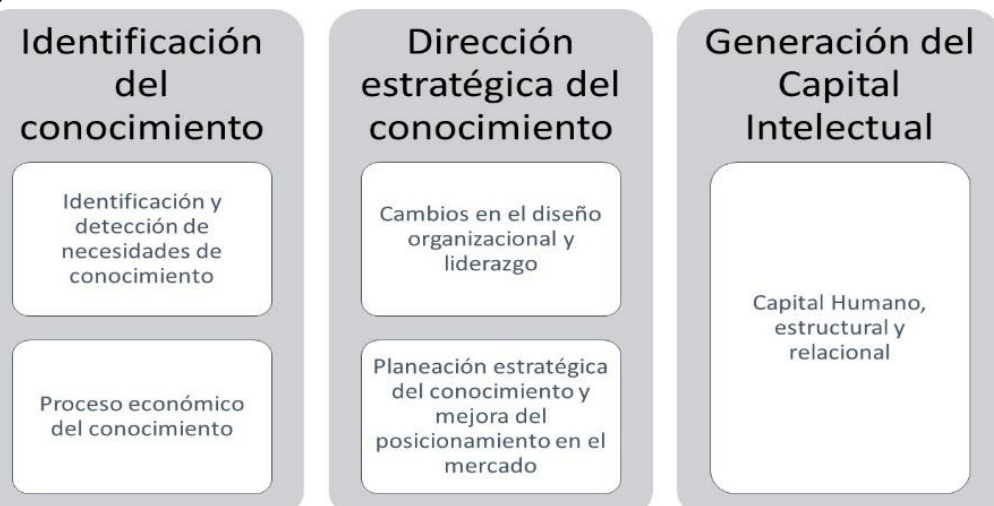

Figura 4. Modelo de gestión del conocimiento para universidades públicas estatales.

Primera etapa. Consiste en que los directivos de las universidades públicas estatales identifiquen el conocimiento que existe en la organización y el que necesitan para definir sus objetivos, además de la identificación de 
prácticas administrativas que es necesario cambiar. También es importante recabar información sobre el proceso económico del conocimiento como son: la producción intercambio, distribución y consumo del mismo.

Segunda etapa. Realizar los cambios en la dirección estratégica del conocimiento para implementar su gestión gerencial.

Tercera etapa. Llevar a cabo la implementación de las estrategias y herramientas para la generación de capital intelectual que incremente el valor de mercado de estas instituciones.

\section{Conclusión}

Para incrementar la productividad del factor conocimiento dentro de las universidades públicas es necesario: identificar el conocimiento que a través de estas instituciones se generan y los que requieren producir para definir sus objetivos de gestión. Es necesario realizar un análisis y la evaluación de las prácticas de gestión que se deben desaprender en estas instituciones. Se debe de impulsar la producción o generación del conocimiento mediante el proceso sinérgico entre el conocimiento tácito y explícito de las IES a través de la realización de sus actividades sustantivas, como son: la docencia, la investigación, la vinculación y la extensión.

Después de producir o generar el conocimiento, es importante planificar su distribución para lograr un impacto positivo en la sociedad. Este conocimiento generado se necesita intercambiar y aplicar, para lo cual es relevante la inversión, promoción y uso de las tecnologías de información y comunicación.

En las universidades públicas se debe de reconocer al conocimiento como un factor más de la producción, ya que de acuerdo con esta investigación, el primer paso para gestionar el conocimiento es que se comprenda la importancia de eficientar su proceso de gestión, al igual que los demás factores: tierra, trabajo, capital y organización.

También, se deben hacer cambios en la estructura organizativa y el liderazgo de estas instituciones, así como una planificación estratégica de los objetivos de gestión del conocimiento. Además, del diseño de estrategias para mejorar su posicionamiento en el mercado educativo.

Para que la gestión del conocimiento sea exitosa, es necesario investigar cómo se produce el conocimiento, ya que esto permitirá elevar su productividad, la forma en que se distribuye, así como la manera en que se intercambia. Es necesario mejorar los canales donde se socializa este factor y el modo en que se aplica para buscar el mejor medio para que los conocimientos lleguen a donde sean útiles y pertinentes.

Cuando las instituciones educativas reflexionen sobre la importancia del factor conocimiento, comprenderán que es necesario utilizarlo en beneficio de la sociedad, ya que la riqueza de las naciones depende de maximizar la 
riqueza de los factores de la producción y en estas instituciones no se potencializa este factor.

Para administrar eficientemente el conocimiento, se requiere evolucionar a la escuela inteligente, donde su principal reto será convertirse en instituciones que aprendan y donde el conocimiento es un factor de relevancia. La escuela inteligente va a requerir del rediseño de su estructura organizativa, de una dirección del conocimiento estratégica y de una medición de su capital intelectual.

Debido a que esta investigación es de carácter exploratorio descriptivo, se recomienda que en las universidades públicas se promuevan líneas de investigación, que aporten conocimientos para el diseño de la estructura organizativa de una universidad inteligente, implementar una dirección del conocimiento estratégica e investigar un sistema de indicadores para medir el capital intelectual, entre otros proyectos. Existen muchas oportunidades para investigar que pueden ayudar a promocionar la universidad inteligente, donde, el factor clave es el conocimiento.

\section{References:}

1. Blázquez, F. (2001). La sociedad de la información y la comunicación. Reflexiones desde la educación. Mérida, España: Junta de Extremadura. Consejería de educación, ciencia y tecnología. Dirección general de ordenación, renovación y Centros. Recuperado de http://www.ub.edu/prometheus21/articulos/obsciberprome/blanquez. pdf.

2. Barroso, F. (2011). Gestión del conocimiento científico en empresas, organizaciones productivas sociales, instituciones de educación superior y centros de investigación en el estado de Yucatán. Ponencia presentada en el Seminario de Investigación de la Facultad de Contaduría y Administración de la UNAM. México, D.F., junio 15.

3. Bustos, E., Cerecedo, M. y Garcia, M. (2014). Desafios de la gestión del Conocimiento Administrativo para el posgrado de Instituciones de Educación Superior Públicas. México: Sociedad Cooperativa de Producción Taller Abierto S.C.L. pp.97

4. Estudio Comparativo de las Universidades Mexicanas (ExeCum). (2014). Explorador de Datos 2014. Recuperado en http://www.execum.unam.mx/

5. Magaña, E., Aguilar, N., Surdez, E. y Quijano, R. (2013). “Gestión del conocimiento en grupos de investigación en ciencias sociales: Caso Universidad Juárez Autónoma de Tabasco, México”. Revista Internacional Administración \& Finanzas. (No.6, Vol. 5, pp. 75-93). Recuperado de http://ssrn.com/abstract=2158865. 
6. Mijangos, J.; Manzo, K. (2012). "Gestión del conocimiento de tres cuerpos académicos consolidados del área educativa”. Revista Electrónica Sinéctica. (Sin No. pp. 1-13). Recuperado en http://www.redalyc.org/pdf/998/99824765003.pdf.

7. Montiel, A. (2015). Historia y aplicaciones de la gestión de conocimiento en educación. Foros de Encuentro Virtual Educa 2014. Lima, Perú. Recuperado de http://reposital.cuaed.unam.mx:8080/jspui/bitstream/123456789/4085 /1/VE14.121.pdf

8. Núñez, Y. y Rodríguez, C. (2015). “Gestión de recursos intangibles en Instituciones de Educación Superior”. Revista de Administración de Empresas. (No. 55, Vo.1, pp. 65-77. Recuperado de https://dx.doi.org/10.1590/S0034-759020150107.

9. Núñez, Y., Arutimuño, M. y Rodríguez, C. (2014). Recursos Intangibles: Sistema de Jerarquización para Instituciones de Educación Superior. 12th Latin American and Caribbean Conference for Engineering and Technology. Guayaquil, Ecuador.

10. Olvera, F. y Morales, J. (2011). "Las instituciones de educación superior organizaciones generadoras del trabajo del conocimiento". Administración y Organizaciones. (No. 26, Vol.1, pp.81-105. Recuperado de http://bidi.xoc.uam.mx/tabla_contenido_fasciculo.php?id_fasciculo= $\underline{578}$.

11. Peñaloza, M., Arévalo, F. y Pirela V. (2010). “Gestión del Capital Intelectual y Perfil de competencias del Personal Directivo de las Universidades Públicas”. Encuentro Educacional. (No.17, Vol.1, pp.138-15). Recuperado de http://www.produccioncientifica.luz.edu.ve/index.php/encuentro/artic le/view/1174.

12. Rivero, D., Vega, V. y Ballagué, J. (2005). "La medición del capital intelectual en las universidades. Un modelo para potenciar su aportación a la sociedad". Capital humano., 185 (1). 30-36. Recuperado de http://www.factorhuma.org/attachments_secure/article/4215/c64_C_ Humano_capital_intelectual_universidades_185_2.pdf

13. Rodríguez-Ponce, E., Pedraza-Rejas, L., Delgado, M. y RodríguezPonce, J. (2010). "Gestión del conocimiento, liderazgo, diseño e implementación de la estrategia: Un estudio empírico en pequeñas y medianas empresas". Ingeniere. Revista Chilena de Ingeniería. (No.18. Vol.3, pp. 373-382). doi: 10.4067/S071833052010000300011 . 
14. Secretaría de Educación Pública (SEP). (2013). Instituciones de Educación Superior. en http://www.ses.sep.gob.mx/instituciones-de-educacion-superior.

15. Topete, C. y Bustos, E. (2008). Sociedad del Conocimiento y Gestión del Capital Intelectual en Instituciones de Educación Superior Públicas Mexicanas. México: Sociedad Cooperativa de Producción Taller Abierto, S.C.L. pp. 195

16. Villaseñor, I, Moreno, C. y Flores, J. (2015). Perspectivas sobre los rankings mundiales de universidades. Revista de la Educación Superior. (No. XLIV, Vol.3, pp. 41-67. Recuperado en http://publicaciones.anuies.mx/pdfs/revista/Revista175_S3A2ES.pdf. 